\title{
A facile strategy for the reduction of graphene oxide and its effect on thermal conductivity of epoxy based composites
}

\author{
F. Xie, S. H. Qi ${ }^{*}, D . W u$ \\ Department of Applied Chemistry, School of Science, Northwestern Polytechnical University, 710072 Xi'an, China
}

Received 16 October 2015; accepted in revised form 30 December 2015

\begin{abstract}
A facile and efficient approach to reduce graphene oxide with Al particles and potassium hydroxide was developed at moderate temperature and the graphene/epoxy composite was prepared by mould casting method. The as-prepared graphene has been confirmed by Transmission electron microscopy, Fourier transform infrared spectrometer, Raman spectroscopy, X-ray diffraction, X-ray photoelectron spectroscopy and Thermal gravimetric analysis. This provides a new green way to synthesize graphene with high surface area and opens another opportunity for the production of graphene. Effects of graphene on thermal conductivity, thermal stability and microstructures of the epoxy-based composite were also investigated. The results showed that thermal conductivity of the composite exhibited a remarkable improvement with increasing content of graphene and thermal conductivity could reach $1.192 \mathrm{~W} /(\mathrm{m} \cdot \mathrm{K})$ when filled with $3 \mathrm{wt} \%$ graphene. Moreover, graphene/epoxy composite exhibits good thermal stability with $3 \mathrm{wt} \%$ graphene.
\end{abstract}

Keywords: polymer composites, oxidation, graphene, graphene oxide, thermal conductivity

\section{Introduction}

Epoxy resin has gained broad attention in various occasions such as printed circuit board, heat exchanger, thermal interface material [1] due to its good adhesion, corrosion resistance, mechanical properties, chemical stability, electrical insulation, low shrinkage and easy processing etc. [2, 3]. However, brittleness $[4,5]$ and low intrinsic thermal conductivity of epoxy based composites limit their potential applications. With further miniaturization of microelectronics, thermal conductivity of polymer material could not meet the requirement of desirable thermal conductance in some special cases [6]. Moreover, integration of transistors has resulted in the escalation of power dissipation as well as an increase in heat flux on the devices. In general, the lifespan of high performance device depends on the heat dissipation capability to some extent $[7,8]$. Therefore, it is essential for the heat generated from the devices to dissipate as effectively as possible. Many ceramic fillers with high thermal conductivity, such as aluminum nitride [9-12], alumina [13, 14], boron nitride [15] and silicon nitride [16] have been reported to improve the thermal conductivity of polymers. Although many researches have been focused on this issue, it still remains unsolved plus some restrictions such as high loading amount and the poor adhesion between filler and matrix.

Graphene emerges as a fascinating material in many technical applications like electronic [17], capacitor [18] and sensor [19] due to its extraordinary electric, thermal and mechanical properties and can be a desirable candidate as thermally conductive filler. Many different approaches for the preparation of graphene have been reported [20-22]. However, the large-scale fabrication of graphene is still a great challenge, which restricts its further applications. Kim et al. [20] reported a large-scale pattern growth

\footnotetext{
${ }^{*}$ Corresponding author, e-mail: qishuhuanwpu@163.com (C) BME-PT
} 
of graphene films for stretchable transparent electrodes via chemical vapor deposition (CVD) on thin nickel layers and $\mathrm{Li}$ [21] grew large-area graphene on copper substrates by CVD using methane. However these approaches have not been expanded broadly because of the high cost and complicated preparation technology. Chemical reduction [23, 24] of graphene oxide (OG) is also considered as an efficient technique to produce graphene on a large scale in spite of its unclear mechanism. Unfortunately, commonly used chemical method is complicated and the reducing agents such as hydrazine [25], sodium borohydride [26], hydroquinone and dimethylhydrazine [27] are hazardous. Besides, some non-carbon impurities can be inevitably introduced into the graphene due to the remaining oxygen atoms. Therefore, a green and efficient method to produce graphene is urgently needed.

Herein, a facile approach is developed to get reduced graphene oxide (RGO) by chemical reduction of OG, which can be conducted using aluminum powder as the reducing agent under alkaline conditions. This approach provides enhanced reducing strength compared with the previous work of Xiaobin [28] and Zhuangjun [29]. Moreover, the obtained graphene suspension is stable within several weeks. Effects of graphene on thermal conductivity, thermal stability and microstructures of the RGO/epoxy composite were also investigated.

\section{Experimental}

\subsection{Materials}

As-received purified natural graphite was supplied by Qingdao Zhong Tian Company. Epoxy resin utilized in this study was a nominally difunctional epoxy resin, bisphenol-A glycidol ether epoxy resin (DGEBA) with an epoxy equivalent of 196 and an average molecular weight of 300 to 400 , which was supplied by Lan Xing New Material Wuxi Resin Co. 2-ethyl-4-methylimidazole (EMI-2, 4) served as a curing agent and was provided by Sino pharm Chemical Reagent Co., Ltd. Acetone, potassium hydroxide $(\mathrm{KOH})$ and hydrochloric acid $(\mathrm{HCl})$ were received from Tianjin $\mathrm{Fu} \mathrm{Yu}$ Fine Chemical Co., Ltd. Concentrated sulfuric acid $\left(\mathrm{H}_{2} \mathrm{SO}_{4}, \mathrm{AR}\right.$, 96 98\%) was purchased from Daxing District Industrial. Potassium permanganate $\left(\mathrm{KMnO}_{4}\right)$ and hydrogen peroxide $\left(\mathrm{H}_{2} \mathrm{O}_{2}\right)$ were supplied by West Long Chemical Co., Ltd. Shantou. Aluminum powder with a diameter of $8 \sim 10 \mu \mathrm{m}$ was purchased from Henan Yuanyang Aluminum industry Co., Ltd. The reagents and materials were directly used without further purification unless otherwise indicated.

\subsection{Chemical reduction of $O G$}

The OG was prepared by modified Hummers' method [30,31] with purified natural graphite. Typically, OG suspension was obtained by dispersing the as-prepared OG in distilled water with a concentration of $2 \mathrm{mg} / \mathrm{mL}$ under intensive sonication for $1 \mathrm{~h}$. The reduction was carried out in water bath at $70^{\circ} \mathrm{C}$ for $30 \mathrm{~min}$ with mild sonication by adding $5 \mathrm{mg}$ aluminum powder and $6 \mathrm{M}$ potassium hydroxide. Subsequently, the original brown suspension turned black after heated to the desired temperature. Finally, $0.5 \mathrm{M}$ hydrochloric acid was added to neutralize excess alkali. The schematic illustration of the reduction procedure is shown in Figure 1.

\subsection{Preparation of RGO/epoxy composites}

The as-prepared RGO was dispersed in acetone under an ultra sonicator bath for $30 \mathrm{~min}$. The dispersion was mixed with DGEBA and the ratio of RGO/DGEBA mixture was adjusted for different content of RGO. The mixture was placed in a warm sonicator bath at $65^{\circ} \mathrm{C}$ until most of the solvents were removed. Subsequently, appropriate amount of curing agent was added. The mixture was stirred for a while to get good homogeneity and accelerate the evaporation of acetone, then placed at $60^{\circ} \mathrm{C}$ for continuous vacuum in order to completely remove the solvent and get rid of air bubbles. Finally, the mixture was slowly poured into a mould and the whole system was placed in an oven. The RGO/epoxy composites were fabricated by curing the mixture at $70^{\circ} \mathrm{C}$ for $3 \mathrm{~h}$, then $110^{\circ} \mathrm{C}$ for $1 \mathrm{~h}$ and at $150^{\circ} \mathrm{C}$ for $3 \mathrm{~h}$.

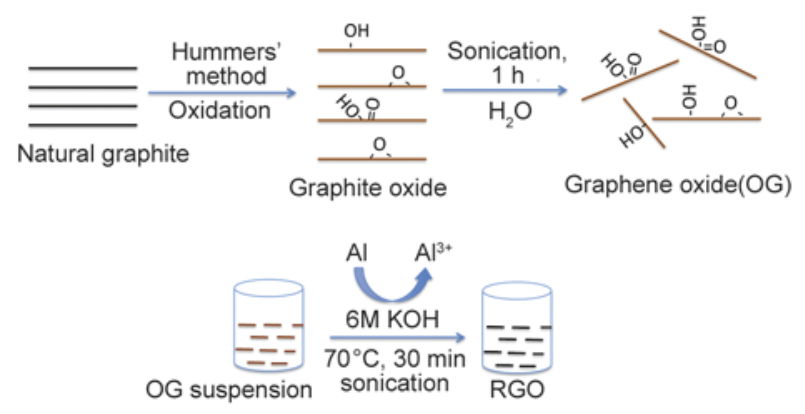

Figure 1. Schematic illustration of the preparation of RGO under alkali condition 


\subsection{Instrumental analysis}

Infrared Spectra were obtained using a WQF-310 type Fourier transform infrared (FT-IR) spectrometer manufactured by Beijing Second Optical Instrument Factory. The RGO samples were ground into powder, and then made into $\mathrm{KBr}$ tablet. The spectrum was collected in a range from 4000 to $400 \mathrm{~cm}^{-1}$. The resolution of apparatus was $4 \mathrm{~cm}^{-1}$ and the number of scans was 16 times. The as-prepared samples were characterized by Raman spectra (HR 800) and X-ray photoelectron spectra (XPS; PHI5300, PerkinElmer) for structure characterization and element

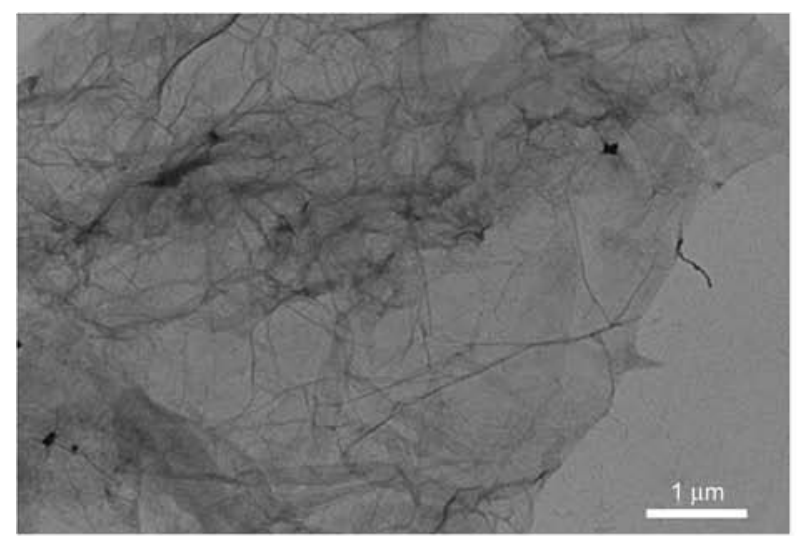

a)

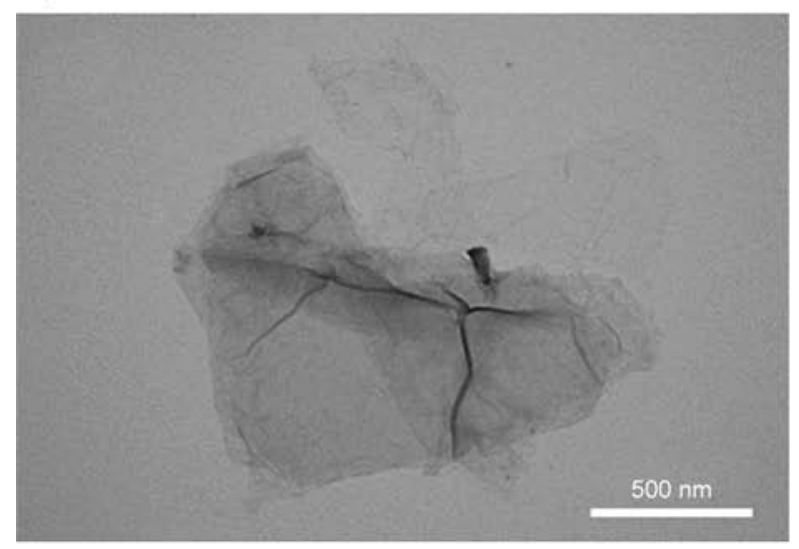

c) analysis. The phase structures of OG and RGO were detected by X-ray powder diffraction (XRD; X'Pert Pro model, Holland) with a scan rate of $5 \% \mathrm{~min}$. The X-ray patterns from 10 to $80^{\circ}$ were obtained. Transmission electron microscopy (TEM) was taken on H800-type (Hitachi Co.) to characterize the microstructures of OG and RGO. Thermal conductivity was conducted by employing a Hot Disk thermal analyzer (Hot Disk AB, Uppsala/Sweden) using transient place source (TPS) method. A disk-shaped TPS sensor with a diameter of $7 \mathrm{~mm}$ and a thickness of $0.07 \mathrm{~mm}$ was placed between two circular sam-

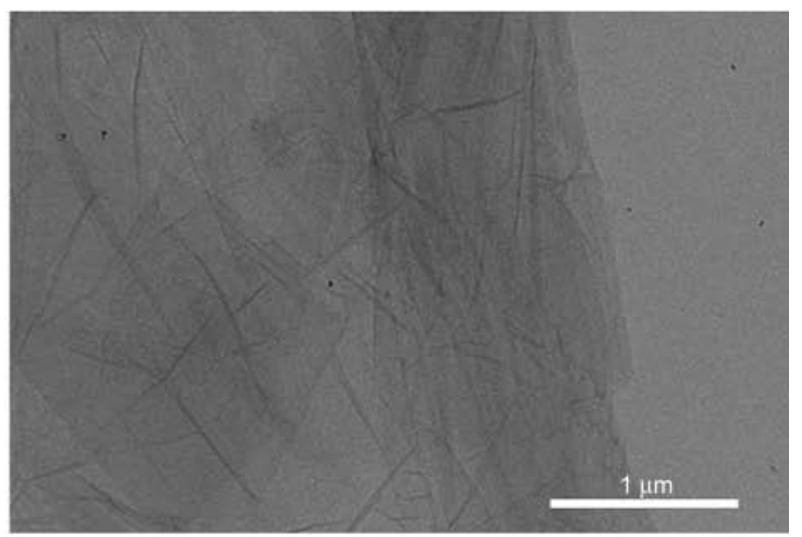

b)

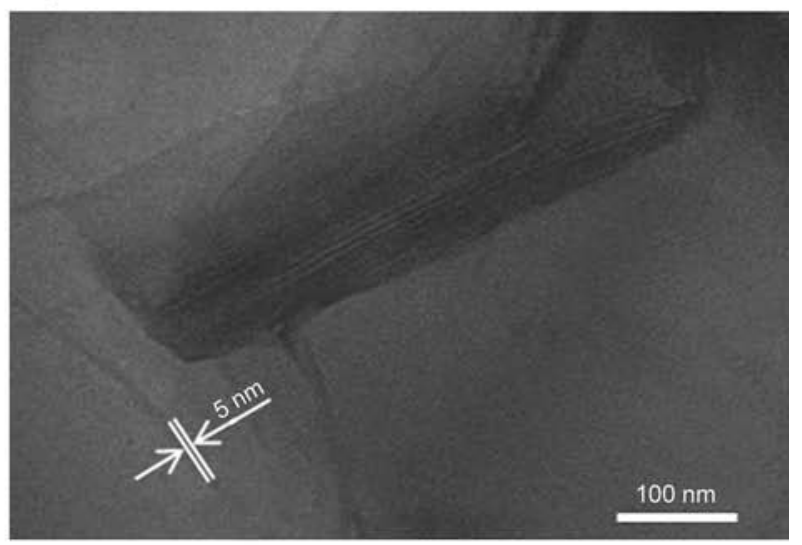

d)

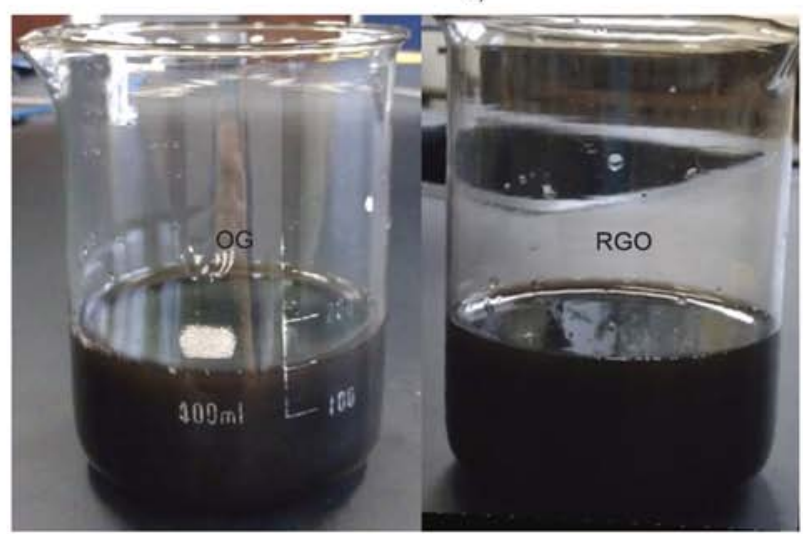

e)

Figure 2. TEM micrographs of RGO under different magnification (a $\sim$ d). Photographs of OG and RGO dispersion solution (e) 
ple pieces with diameters of $20 \mathrm{~mm}$ and thicknesses of $2 \mathrm{~mm}$. The upper surface and lower surface were polished with fine sand paper prior to use. The morphology was observed using a field emission scanning electron microscope (FESEM) (JSM-6360LV, Japan).

\section{Results and discussion}

\subsection{TEM analysis of RGO}

TEM images of RGO under different magnification are shown in Figure 2a, 2d). It can be observed from Figures $2 \mathrm{a}$ and $2 \mathrm{~b}$ that the crumpled silk waves of graphene sheets possess large surface areas. Some graphene sheets fold and crinkle at some places, which can be seen in Figures $2 \mathrm{c}$ and $2 \mathrm{~d}$. Moreover, the as-prepared RGO is mostly monolayer while some multiple-layered graphene also exist, the thickness of which ranges from 5 to $50 \mathrm{~nm}$ (Figure 2d). The multiple-layered graphene should also exfoliate to monolayer as it experiences further ultrasonic treatment and agitation during the preparation. During the reduction process, a large amount of hydrogen bubbles were produced and the yellow-brown graphite oxide suspension gradually turned black, which are indicative of successfully deoxygenation of graphite oxide. The as-prepared RGO is very sta-

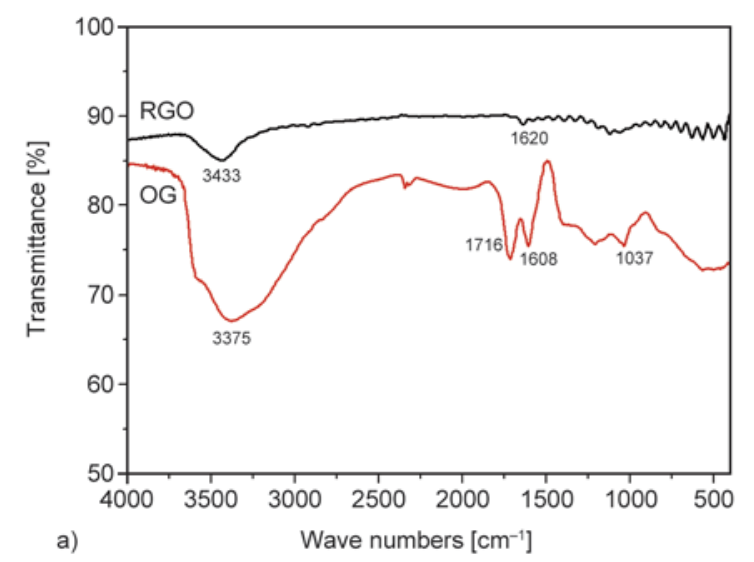

ble in water within several weeks, which can be seen in Figure 2e.

\subsection{Structural characterization of OG and RGO}

FTIR spectra shown in Figure 3a confirm the oxidation of graphite and identify specific functional group indicating the successful reduction of OG. A broad and intense peak centered at $3375 \mathrm{~cm}^{-1}$ from the spectrum of $\mathrm{OG}$ is attributed to the stretching vibration of -OH group. The peak around $1608 \mathrm{~cm}^{-1}$ is associated with vibration of absorbed water molecule [32] and also somewhat attributed to the skeletal vibrations of non-oxidized graphitic domains [33]. The characteristic peak at $1716 \mathrm{~cm}^{-1}$ is attributed to the stretching vibration of $\mathrm{C}=\mathrm{O}$ bond in $-\mathrm{COOH}$ group. The signal at $1037 \mathrm{~cm}^{-1}$ is assigned to the stretching vibration absorption of the $\mathrm{C}-\mathrm{O}-\mathrm{C}$ bond. After deoxidization, the characteristic peaks of $\mathrm{C}=\mathrm{O}$ bond and $\mathrm{C}-\mathrm{O}-\mathrm{C}$ bond disappeared. Moreover, the absorption peak of $-\mathrm{OH}$ group became weak and narrow and the peak around $1620 \mathrm{~cm}^{-1}$ decreased, indicating most of the oxygen-containing functional groups of OG were successfully deoxidized.

The structural changes of $O G$ after reduction can also be reflected in the Raman spectra shown in Fig-

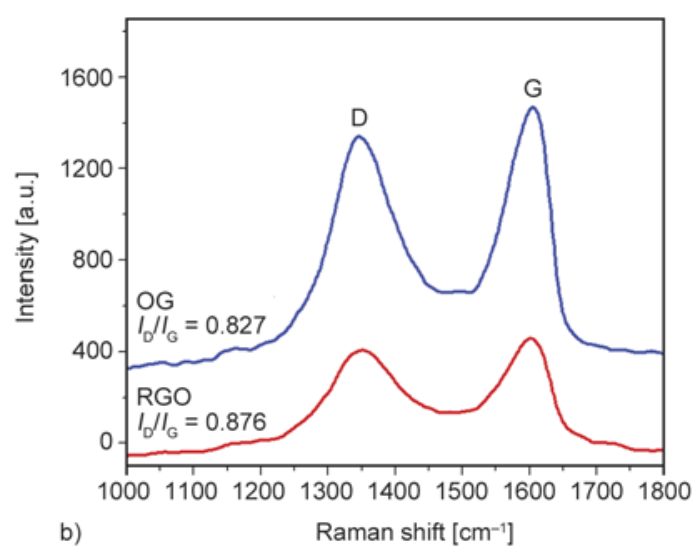

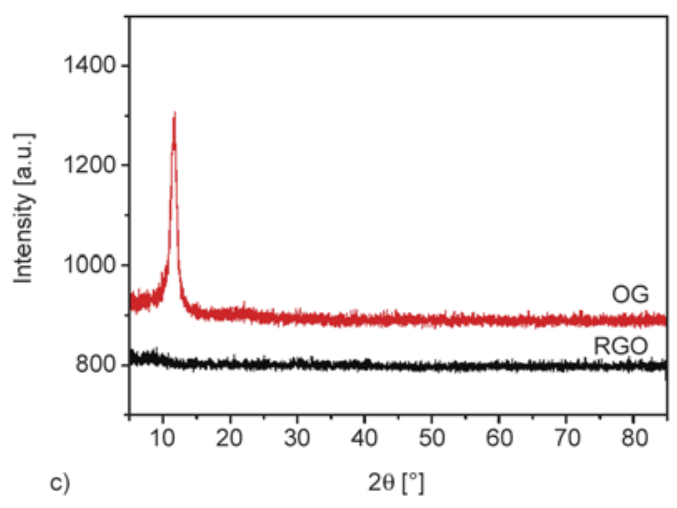

Figure 3. FT-IR spectra (a), Raman spectra (b), XRD patterns (c) of OG and RGO 
ure $3 \mathrm{~b} . I_{\mathrm{D}} / I_{\mathrm{G}}$ ratio can be used to qualitatively characterize the change of defects in the carbon materials. The intensity ratio $\left(I_{\mathrm{D}} / I_{\mathrm{G}}\right)$ for OG was slightly increased after reduction. The reason may be that a decrease in the average size of the $\mathrm{sp}^{2}$ domains upon the reduction of $\mathrm{OG}$, indicating the formation of some $\mathrm{sp}^{3}$ carbon after acid oxidation. The underlying mechanism is still unclear. The intensity ratio $\left(I_{\mathrm{D}} / I_{\mathrm{G}}\right)$ of the $D$ bond and $G$ bond of OG is about 0.827 , while the ratio of RGO is 0.876 . This $I_{\mathrm{D}} / I_{\mathrm{G}}$ ratio is consistent with the results reported by other researchers [34]. $D$ band of RGO becomes stronger and broader, indicating that a higher level of disorder of the graphene layers and defects were introduced. XRD is used to further study the changes in structure. The XRD patterns shown in Figure $3 \mathrm{c}$ indicate that $\mathrm{OG}$ has a larger interlayer distance $(2 \theta=$ 10.3) from (001) due to the formation of hydroxyl, epoxy, and carboxyl groups. After reduction, the interlayer distance decreases due to the removal of some oxygen-containing functional groups, which means that the $\mathrm{sp}^{2}$ carbon is reestablished during the reduction. This can be explained by the ring-opening reaction of the epoxides. The complete disappearance of the native graphite XRD peaks in the
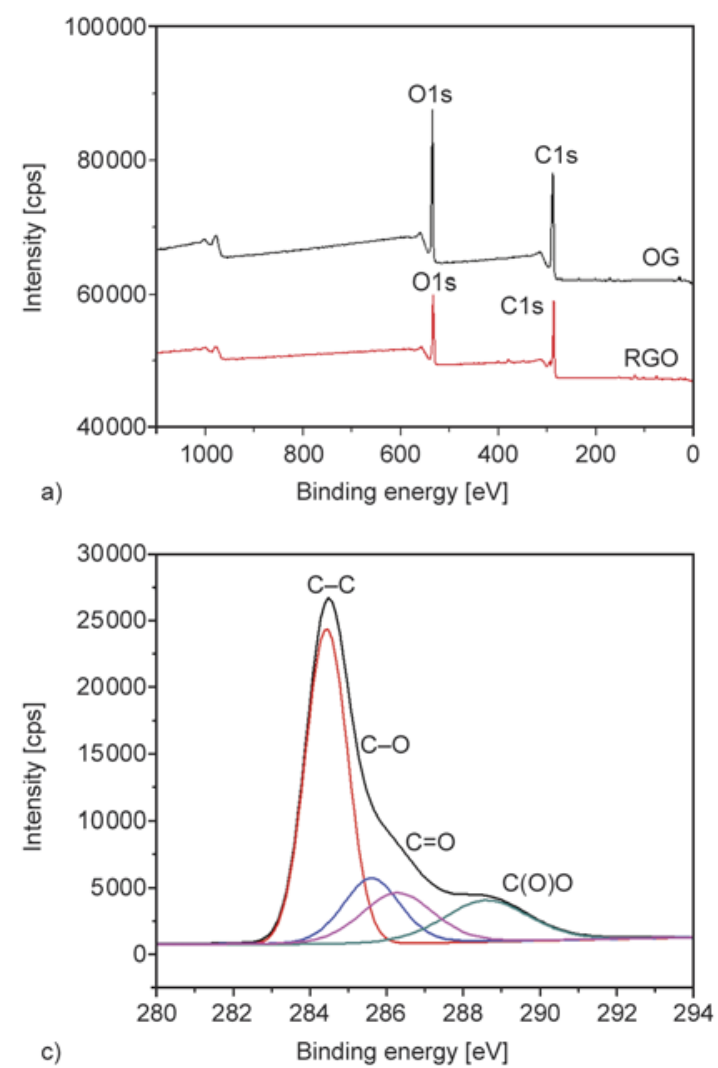

pattern of the obtained graphene supports its single sheet nature.

\subsection{XPS characterization and TGA analysis}

The chemical compositions of OG and RGO were investigated by XPS. The XPS survey is presented in Figure 4a and high-resolution C1s spectra of OG and RGO were shown in Figure $4 b$ and Figure 4c, respectively. The survey clearly revealed that the intensity of O1s peak of RGO decreased obviously as compared to that of $\mathrm{OG}$, suggesting the ratio of $\mathrm{C} / \mathrm{O}$ increased remarkably after chemical reduction. That is to say, the majority of oxygen-containing functional groups were successfully removed. The increased $\mathrm{C}=\mathrm{C}$ peak at $284.8 \mathrm{eV}$ and decreased signal intensity of $\mathrm{C}-\mathrm{O}$ at $286.9 \mathrm{eV}$ in $\mathrm{C} 1 \mathrm{~s}$ XPS spectrum of RGO further indicate the reduction of graphite oxide and formation of graphene.

Thermal stabilities of OG and RGO are investigated by TGA and the result is shown in Figure 4d. OG is thermally unstable and starts to lose mass upon heating even below $100^{\circ} \mathrm{C}$. There is a sharp weight loss at approximately $200^{\circ} \mathrm{C}$, which is caused by the decomposition of oxygen-containing functional groups into $\mathrm{CO}, \mathrm{CO}_{2}$, water vapor and so on. Hence,
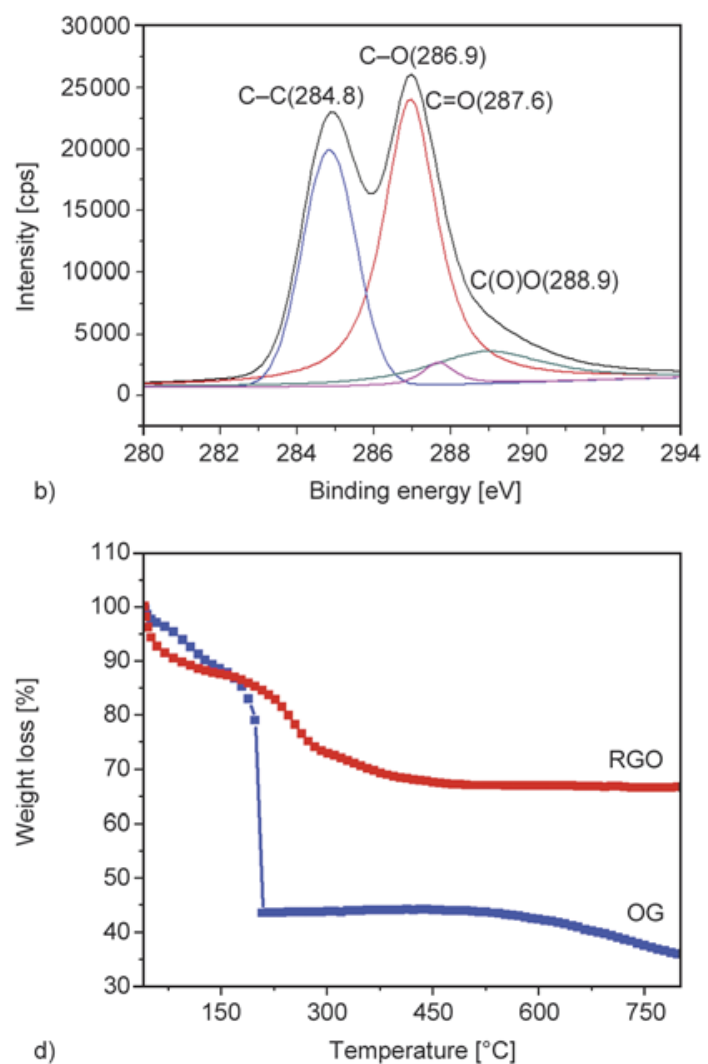

Figure 4. XPS spectra and TGA curves of OG and RGO. XPS Survey (a), high resolution C1s region of OG (b) and RGO (c), TGA curves (d). 
the thermal decomposition of $\mathrm{OG}$ is accompanied by a vigorous release of gas, resulting in a rapid thermal expansion of the material, which is revealed in the curve. After reduction, thermal stability of RGO increased due to the removal of thermally labile oxygen-containing functional groups, indicative of the successful reduction process. Besides, no significant weight loss can be found after heated up to $800^{\circ} \mathrm{C}$.

\subsection{Thermal properties and microstructure of RGO/epoxy composites}

Figure 5a shows that with the increasing content of $\mathrm{RGO}$, there is a significant increase in thermal conductivity of the RGO/epoxy composites. This is because that adding the fillers could form a thermal conductive network path. Furthermore, higher filler content could form more conductive paths thus increasing the heat flow of the surface $[35,36]$. Thermal conductivity is affected by the filler structure, loading, and dispersion. Besides, the interfacial physical contact between polymer and filler is also very critical since phonons are very sensitive to surface defects. Previously, we did some research on the amino-functionalized MWCNT/epoxy composites
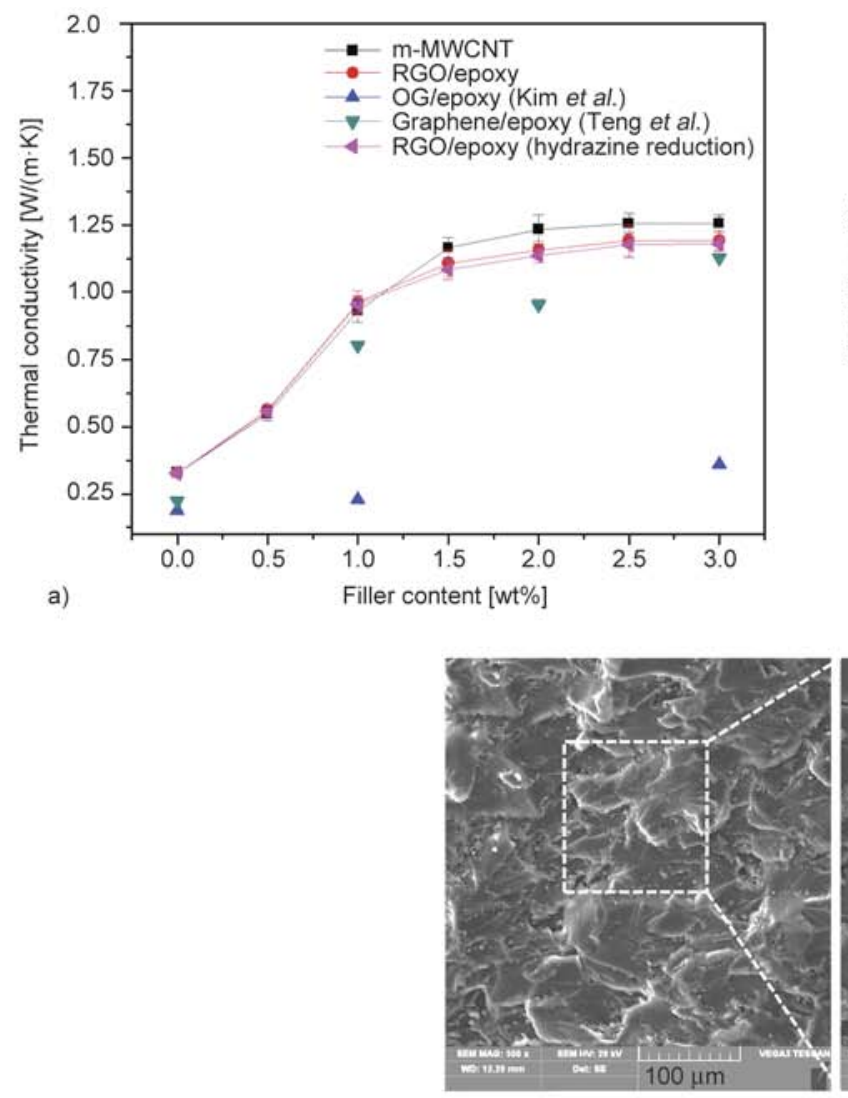

c) in order to decrease the interfacial barrier [37]. The thermal conductivity of MWCNT/epoxy composites is significantly increased. However, the process is a little more complicated than our method reported here. The as-prepared RGO possesses higher surface area and can connect with the epoxy adequately, which reduces the barriers of phonon transport to a large extent. Moreover, the excellent solubility of RGO facilitates its dispersion in polymer composites, resulting in an increased contact surface area between RGO and epoxy matrix. Accordingly, it can facilitate the heat flows and promote phonon diffusion in RGO/epoxy composites.

As can be seen in Figure 5a, thermal conductivity of $\mathrm{RGO} /$ epoxy composite is almost the same as that of amino-functionalized MWCNT/epoxy composite. By contrast, thermal conductivity of composite with RGO by common hydrazine reduction was a little bit lower than that of RGO/epoxy composite acquired by our method, which is also shown in Figure 5a. RGO/epoxy composites prepared by this method exhibit the best efficiency in improving thermal conductivity, compared with those filled with ordinary graphene or OG [38]. It was noticeable that thermal conductivity of RGO/epoxy composite increased
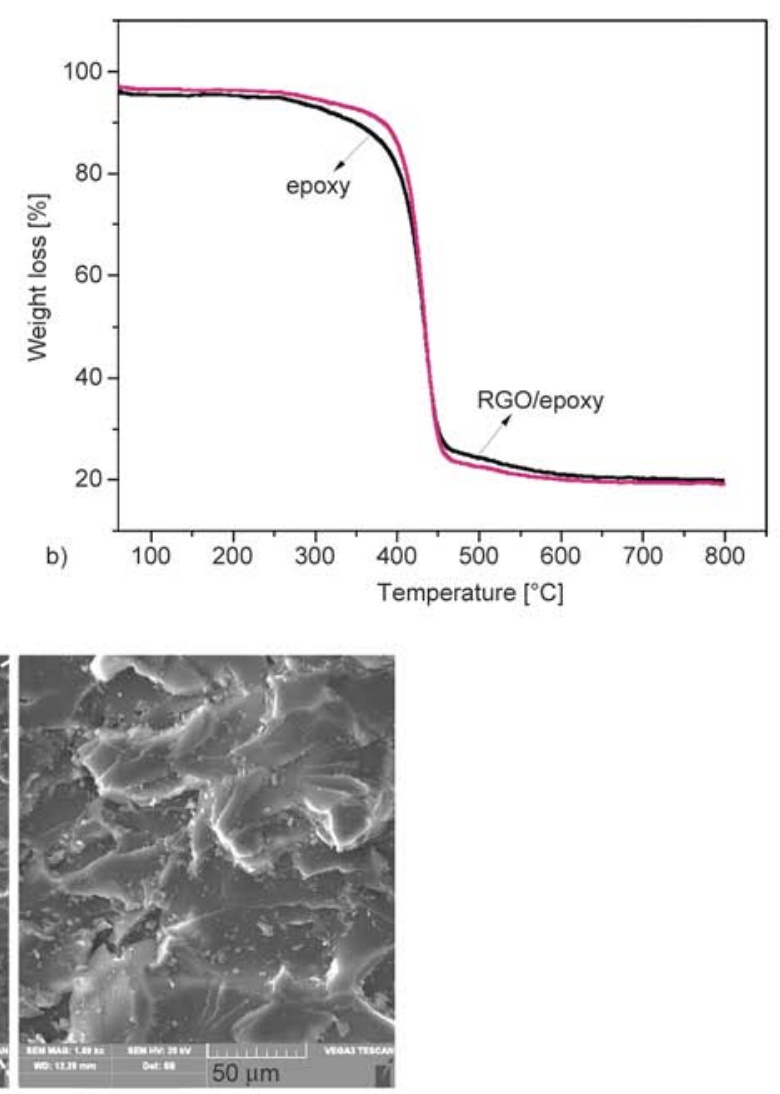

Figure 5. Thermal conductivity (a), thermal stability (b), and cross-section images (c) of epoxy-based composites 
significantly and reached $1.192 \mathrm{~W} /(\mathrm{m} \cdot \mathrm{K})$ when filled with $3 \mathrm{wt} \%$ RGO, higher than that of OG/epoxy with the same weight percentage (Figure 5a) by Kim et al. [39]. In general, 40 50 $\mathrm{wt} \%$ ceramic fillers is required to achieve the comparable thermal conductivity. As is also indicated in Figure 5a, the thermal conductivity of epoxy resin system increases obviously with increasing filler content up to $1 \mathrm{wt} \%$. After that, it tends to increase slower. This can be explained by the established percolation theory [40].

Thermal stability of RGO/epoxy composite with a filler content of $3 \mathrm{wt} \%$ is shown in Figure $5 \mathrm{~b}$. The initial thermal decomposition temperature of pure epoxy is $348^{\circ} \mathrm{C}$. Compared to the pure epoxy resin, the initial thermal decomposition temperature of RGO/epoxy composite increased about $40^{\circ} \mathrm{C}$. Thermal stability of RGO/epoxy composite not only depends on thermal stability of RGO itself, but also relates to the dispersion of RGO in matrix and the interaction between them. If agglomeration of RGO exists, the relatively poor dispersion could make some defects in the epoxy matrix, which will reduce the initial decomposition temperature of the composites. Besides, the good interfacial affinity between RGO and matrix could delay the volatilization of small molecules at high temperature, thus increasing the thermal stability of the composites.

Cross-section SEM images of the RGO/epoxy composite can be seen in Figure $5 \mathrm{c}$. No aggregations can be observed in the SEM images, indicating that RGO is uniformly dispersed in the epoxy resin. RGO is not easily observed in epoxy matrix even under higher magnification, while cracks in cross section of the composites verified that the adhesion between the filler and matrix is strong enough. The cross section of pure epoxy should be smooth and the crack is uniform and orderly [39]. The introduction of RGO can prevent rapid expansion of macroscopic crack in matrix and cracks become disordered and consume a part of the energy when subjected to external forces. Overall, the as-prepared RGO can homogeneously disperse in the epoxy matrix and the results show good interfacial affinity between RGO and epoxy matrix, which are consistent with the results of the thermal conductivity.

\section{Conclusions}

In conclusion, the exfoliated OG undergoes quick de-oxygenation process by aluminum in alkali solutions at moderate temperature. This provides a new facile way to synthesize graphene with large surface area and opens another opportunity for the production of graphene. Results from TEM, FT-IR, Raman, XRD, XPS and TGA analysis demonstrate that RGO is successfully prepared by this new method, although the reduction mechanism is still not clear enough. What's more, RGO/epoxy composite exhibits remarkable improvement of thermal conductivity. The thermal conductivity of RGO/ epoxy composite increased significantly and reached $1.192 \mathrm{~W} /(\mathrm{m} \cdot \mathrm{K})$ when filled with $3 \mathrm{wt} \% \mathrm{RGO}$. The initial thermal decomposition temperature of $\mathrm{RGO} /$ epoxy composite is improved compared with pure epoxy resin from TGA analysis and the microstructure images further verify the good interfacial affinity between RGO and epoxy matrix.

\section{Acknowledgements}

The authors would like to express their deepest acknowledgement to Prof. Zhang for her continuous encouragement and help to facilitate this work. Authors also appreciate Northwestern Polytechnical University for its support as well as the companies and relatives who friendly offered the materials and help.

\section{References}

[1] Yu A., Ramesh P., Sun X., Bekyarova E., Itkis M. E., Haddon R. C.: Enhanced thermal conductivity in a hybrid graphite nanoplatelet - Carbon nanotube filler for epoxy composites. Advanced Materials, 20, 47404744 (2008). DOI: $10.1002 /$ adma.200800401

[2] Teng C-C., Ma C-C. M., Chiou K-C., Lee T-M., Shih Y-F.: Synergetic effect of hybrid boron nitride and multi-walled carbon nanotubes on the thermal conductivity of epoxy composites. Materials Chemistry and Physics, 126, 722-728 (2011). DOI: $10.1016 /$ j.matchemphys.2010.12.053

[3] Suriati G., Mariatti M., Azizan A.: Effects of filler shape and size on the properties of silver filled epoxy composite for electronic applications. Journal of Materials Science: Materials in Electronics, 22, 56-63 (2011). DOI: 10.1007/s10854-010-0082-2

[4] Özarslan Ö., Yildiz E., İnan T. Y., Kuyulu A., Güngör A.: Novel amine terminated elastomeric oligomers and their effects on properties of epoxy resins as a toughener. Journal of Applied Polymer Science, 115, 37-45 (2010). DOI: 10.1002/app.29693

[5] Luo Y., Zhang M., Dang G., Li Y., An X., Chen C., Yi $\mathrm{X}$.: Toughening of epoxy resin by poly(ether ether ketone) with pendant fluorocarbon groups. Journal of Applied Polymer Science, 122, 1758-1765 (2011). DOI: 10.1002/app.34292 
[6] Lee G-W., Park M., Kim J., Lee J. I., Yoon H. G.: Enhanced thermal conductivity of polymer composites filled with hybrid filler. Composites Part A: Applied Science and Manufacturing, 37, 727-734 (2006). DOI: 10.1016/j.compositesa.2005.07.006

[7] Sim L. C., Ramanan S. R., Ismail H., Seetharamu K. N., Goh T. J.: Thermal characterization of $\mathrm{Al}_{2} \mathrm{O}_{3}$ and $\mathrm{ZnO}$ reinforced silicone rubber as thermal pads for heat dissipation purposes. Thermochimica Acta, 430, 155-165 (2005). DOI: $10.1016 /$ j.tca.2004.12.024

[8] Zhou W., Qi S., Tu C., Zhao H., Wang C., Kou J.: Effect of the particle size of $\mathrm{Al}_{2} \mathrm{O}_{3}$ on the properties of filled heat-conductive silicone rubber. Journal of Applied Polymer Science, 104, 1312-1318 (2007). DOI: $10.1002 /$ app.25789

[9] Gu J., Zhang Q., Dang J., Zhang J., Yang Z.: Thermal conductivity and mechanical properties of aluminum nitride filled linear low-density polyethylene composites. Polymer Engineering and Science, 49, 1030-1034 (2009). DOI: $10.1002 /$ pen.21336

[10] Peng W., Huang X., Yu J., Jiang P., Liu W.: Electrical and thermophysical properties of epoxy/aluminum nitride nanocomposites: Effects of nanoparticle surface modification. Composites Part A: Applied Science and Manufacturing, 41, 1201-1209 (2010). DOI: $10.1016 /$ j.compositesa.2010.05.002

[11] Bae J-W., Kim W., Cho S-H., Lee S-H.: The properties of AlN-filled epoxy molding compounds by the effects of filler size distribution. Journal of Materials Science, 35, 5907-5913 (2000). DOI: $10.1023 / \mathrm{A}: 1026741300020$

[12] Teng C-C., Ma C-C. M., Chiou K-C., Lee T-M.: Synergetic effect of thermal conductive properties of epoxy composites containing functionalized multi-walled carbon nanotubes and aluminum nitride. Composites Part B: Engineering, 43, 265-271 (2012). DOI: 10.1016/j.compositesb.2011.05.027

[13] Mcgrath L. M., Parnas R. S., King S. H., Schroeder J. L., Fischer D. A., Lenhart J. L.: Investigation of the thermal, mechanical, and fracture properties of aluminaepoxy composites. Polymer, 49, 999-1014 (2008).

DOI: $10.1016 /$ j.polymer.2007.12.014

[14] Im H., Kim J.: Enhancement of the thermal conductivity of aluminum oxide-epoxy terminated poly(dimethyl siloxane) with a metal oxide containing polysiloxane. Journal of Materials Science, 46, 6571-6580 (2011). DOI: 10.1007/s10853-011-5604-7

[15] Zhou W., Qi S., An Q., Zhao H., Liu N.: Thermal conductivity of boron nitride reinforced polyethylene composites. Materials Research Bulletin, 42, 1863-1873 (2007).

DOI: $10.1016 /$ j.materresbull.2006.11.047
[16] He H., Fu R., Shen Y., Han Y., Song X.: Preparation and properties of $\mathrm{Si}_{3} \mathrm{~N}_{4} / \mathrm{PS}$ composites used for electronic packaging. Composites Science and Technology, 67, 2493-2499 (2007).

DOI: 10.1016/j.compscitech.2006.12.014

[17] Eda G., Fanchini G., Chhowalla M.: Large-area ultrathin films of reduced graphene oxide as a transparent and flexible electronic material. Nature Nanotechnology, 3, 270-274 (2008).

DOI: 10.1038/nnano.2008.83

[18] Miller J. R., Outlaw R. A., Holloway B. C.: Graphene double-layer capacitor with ac line-filtering performance. Science, 329, 1637-1639 (2010).

DOI: $10.1126 /$ science. 1194372

[19] Lu C-H., Yang H-H., Zhu C-L., Chen X., Chen G-N.: A graphene platform for sensing biomolecules. Angewandte Chemie, 121, 4879-4881 (2009).

DOI: $10.1002 /$ anie.200901479

[20] Kim K. S., Zhao Y., Jang H., Lee S. Y., Kim J. M., Kim K. S., Ahn J. H., Kim P., Choi J. Y., Hong B. H.: Largescale pattern growth of graphene films for stretchable transparent electrodes. Nature, 457, 706-710 (2009). DOI: 10.1038/nature07719

[21] Li X., Cai W., An J., Kim S., Nah J., Yang D., Piner R., Velamakanni A., Jung I., Tutuc E., Banerjee S. K., Colombo L., Ruoff R. S.: Large-area synthesis of highquality and uniform graphene films on copper foils. Science, 324, 1312-1314 (2009). DOI: $10.1126 /$ science. 1171245

[22] Xu C., Wang X., Zhu J.: Graphene-metal particle nanocomposites. Journal of Physical Chemistry C, 112, 19841-19845 (2008). DOI: $10.1021 / j p 807989 b$

[23] Chen W., Yan L.: In situ self-assembly of mild chemical reduction graphene for three-dimensional architectures. Nanoscale, 3, 3132-3137 (2011). DOI: $10.1039 / \mathrm{c} 1 \mathrm{nr} 10355 \mathrm{e}$

[24] Compton O. C., Dikin D. A., Putz K. W., Brinson L. C., Nguyen S. T.: Electrically conductive 'alkylated' graphene paper via chemical reduction of amine-functionalized graphene oxide paper. Advanced Materials, 22, 892-896 (2010).

DOI: $10.1002 / \mathrm{adma} .200902069$

[25] Park S., An J., Potts J. R., Velamakanni A., Murali S., Ruoff R. S.: Hydrazine-reduction of graphite- and graphene oxide. Carbon, 49, 3019-3023 (2011). DOI: $10.1016 /$ j.carbon.2011.02.071

[26] Shin H-J., Kim K. K., Benayad A., Yoon S-M., Park H. K., Jung I-S., Jin M. H., Jeong H-K., Kim J. M., Choi J-Y., Lee Y. H.: Efficient reduction of graphite oxide by sodium borohydride and its effect on electrical conductance. Advanced Functional Materials, 19, 19871992 (2009).

DOI: $10.1002 / \mathrm{adfm} .200900167$ 
[27] Stankovich S., Dikin D. A., Dommett G. H. B., Kohlhaas K. M., Zimney E. J., Stach E. A., Piner R. D., Nguyen S. T., Ruoff R. S.: Graphene-based composite materials. Nature, 442, 282-286 (2006).

DOI: $10.1038 /$ nature 04969

[28] Fan X., Peng W., Li Y., Li X., Wang S., Zhang G., Zhang F.: Deoxygenation of exfoliated graphite oxide under alkaline conditions: A green route to graphene preparation. Advanced Materials, 20, 4490-4493 (2008). DOI: $10.1002 /$ adma.200801306

[29] Fan Z., Wang K., Wei T., Yan J., Song L., Shao B.: An environmentally friendly and efficient route for the reduction of graphene oxide by aluminum powder. Carbon, 48, 1686-1689 (2010). DOI: $10.1016 /$ j.carbon.2009.12.063

[30] Hummers W. S., Offeman R. E.: Preparation of graphitic oxide. Journal of the American Chemical Society, 80, 1339 (1958). DOI: $10.1021 / \mathrm{ja} 01539 \mathrm{a} 017$

[31] Shen J., Hu Y., Li C., Qin C., Ye M.: Synthesis of amphiphilic graphene nanoplatelets. Small, 5, 82-85 (2009). DOI: $10.1002 / \mathrm{smll} .200800988$

[32] Szabó T., Berkesi O., Dékány I.: Drift study of deuterium-exchanged graphite oxide. Carbon, 43, 31863189 (2005).

DOI: 10.1016/j.carbon.2005.07.013

[33] Chen C., Yang Q-H., Yang Y., Lv W., Wen Y., Hou P-X., Wang M., Cheng H-M.: Self-assembled free-standing graphite oxide membrane. Advanced Materials, 21, 3007-3011 (2009).

DOI: 10.1002/adma.200990138

[34] Yang D., Velamakanni A., Bozoklu G., Park S., Stoller M., Piner R. D., Stankovich S., Jung I., Field D. A., Ventrice C. A., Ruoff R. S.: Chemical analysis of graphene oxide films after heat and chemical treatments by Xray photoelectron and micro-raman spectroscopy. Carbon, 47, 145-152 (2009).

DOI: $10.1016 /$ j.carbon.2008.09.045
[35] Li T-L., Hsu S-L. C.: Enhanced thermal conductivity of polyimide films via a hybrid of micro- and nano-sized boron nitride. Journal of Physical Chemistry B, 114, 6825-6829 (2010).

DOI: $10.1021 / \mathrm{jp} 101857 \mathrm{w}$

[36] Zhou T., Wang X., Liu X., Xiong D.: Improved thermal conductivity of epoxy composites using a hybrid multiwalled carbon nanotube/micro-SiC filler. Carbon, $\mathbf{4 8 ,}$ 1171-1176 (2010).

DOI: $10.1016 /$ j.carbon.2009.11.040

[37] Xie F., Qi S., Yang R., Wu D.: High thermal conductive m-xylylenediamine functionalized multiwall carbon nanotubes/epoxy resin composites. Journal of Applied Polymer Science, 132, 41255/1-41255/6 (2015). DOI: $10.1002 / A P P .41255$

[38] Teng C-C., Ma C-C. M., Lu C-H., Yang S-Y., Lee SH., Hsiao M-C., Yen M-Y., Chiou K-C., Lee T-M.: Thermal conductivity and structure of non-covalent functionalized graphene/epoxy composites. Carbon, 49, 5107-5116 (2011).

DOI: 10.1016/j.carbon.2011.06.095

[39] Kim J., Yim B-S., Kim J-M., Kim J.: The effects of functionalized graphene nanosheets on the thermal and mechanical properties of epoxy composites for anisotropic conductive adhesives (ACAS). Microelectronics Reliability, 52, 595-602 (2012). DOI: $10.1016 /$ j.microrel.2011.11.002

[40] Shante V. K. S., Kirkpatrick S.: An introduction to percolation theory. Advances in Physics, 20, 325-357 (1971).

DOI: $10.1080 / 00018737100101261$ 